\title{
La Autoconfianza en el Deporte en Edad Escolar
}

\author{
Self-Confidence in Sport in School Age
}

\author{
Enrique Fradejas Medrano ${ }^{1}$, María Espada $\operatorname{Mateos}^{2}$ y Rocío Garrido Martos 3
}

\begin{abstract}
Resumen
Este estudio analiza el estado de autoconfianza en el deporte en edad escolar, en función del género, tipo de deporte y edad. El tamaño de la muestra fue de 816 personas (50.3\% varones y $49.7 \%$ mujeres) entre 12 y 18 años, que practican diferentes deportes individuales y colectivos en Castilla-La Mancha. Se utilizó el cuestionario sobre Características Psicológicas relacionadas con el Rendimiento Deportivo (CPRD). Los resultados muestran diferencias estadísticamente significativas en relación con el género, la modalidad deportiva y el tipo de deporte. Efectivamente, se observa un mayor nivel de autoconfianza en los hombres, en el Judo y el Fútbol y en la práctica deportiva individual, en relación con el de las mujeres, otro tipo de deporte y la práctica en deportes colectivos. Respecto de la edad, no se hallaron diferencias estadísticamente significativas. Se discuten los alcances de los resultados y se reflexiona sobre su utilidad en la formación deportiva.
\end{abstract}

Palabras clave: autoconfianza, género, tipo de deporte, edad, deporte escolar

\begin{abstract}
This study analyses self-confidence state in sport in school age regarding gender, type of sport and age. The sample size is 816 people (50.3\% males and $49.7 \%$ females) between the ages of 12 and 18 who practise different individual and collective sports in the Spanish region of Castilla-La Mancha. The given questionnaire is called "Psychological Characteristics related to Sporting Performance" (CPRD). The results have found statistically significant differences in relation to gender, due to men show a higher selfconfidence level; also in relation to the sports modality, because in Judo and Football there are higher selfconfidence levels compared to other analysed sports including Voleyball. Moreover in relation to the type of sport, sportsmen/women who practise individual sports show a higher self-confidence level compared to sportsmen/women who practise team sports. The results have not found statistically significant differences in relation to age.
\end{abstract}

Keywords: self-confidence, gender, type of sport, school age, school sports

\footnotetext{
${ }^{1}$ Doctor en Educación. Profesor del IES Ojos del Guadiana. C/ Francisco Pérez Fernández, 3. 13250. Daimiel. Ciudad Real, España. Tel.: (34)660500771. Correo: enriquefmpm@yahoo.es

${ }^{2}$ Doctora en Ciencias de la Actividad Física y del Deporte. Facultad de Ciencias de la Actividad Física y del Deporte. Universidad Politécnica de Madrid. Calle Martín Fierro, 7, 28040 Madrid, España. Tel.: +34913 364092. Correo: maria.espada@upm.es

${ }^{3}$ Doctora en Educación. Profesora de la Universidad a Distancia de Madrid. Carretera de la Coruña, KM.38,5. Vía de servicio nº 15. 28400 Collado Villalba. Madrid, España. Tel.: (34)918561699. Correo: rocio.garrido@udima.es
} 


\section{Introducción}

Vives y Garcés de los Fayos (2002) revisaron las definiciones sobre autoconfianza que proponen distintos autores (Bandura, 1977; Buceta, 1996; Cruz, 1997; Vealey, 1986; Weinberg \& Gould, 1996) en un intento de unificar sus propuestas. Con ese propósito la definen como la convicción que tiene una persona para poder poner en práctica las habilidades tanto físicas como mentales necesarias para ejecutar con éxito la conducta requerida, con la finalidad de producir un resultado determinado.

Podríamos decir, a nivel general, que la autoconfianza parece depender de la congruencia entre las expectativas previas y los logros deportivos reales. Pero más específicamente, los aspectos que influyen en la autoconfianza son (Bandura, 1977; Buceta, 1999): percepción de control de la situación, perspectiva realista y ajustada de las expectativas, percepción apropiada del éxito-fracaso, trascendencia de la evaluación del rendimiento y experiencias reales de éxito deportivo.

Muchos niños y adolescentes, en el deporte y en otros campos, tienen una autoconfianza débil. Se sienten inseguros antes de afrontar un reto, dudan respecto de su rendimiento y no tienen claro si han respondido a aquello que se esperaba de ellos. En general, son edades en las que la inseguridad suele predominar sobre la autoconfianza dificultando, en muchos casos, el progreso de los deportistas (Buceta, 2004).

Desarrollar pues la autoconfianza de los jóvenes es muy importante en su proceso formativo como deportistas y como personas (Buceta, 2004). Como deportistas, porque la autoconfianza es la clave para que progresen, sean capaces de afrontar satisfactoriamente las situaciones más difíciles de las competiciones y sigan trabajando con la ilusión de alcanzar metas ambiciosas. Como personas, porque confiar en uno mismo ayuda a afrontar las demandas de la vida y contribuye a mejorar el autoconcepto y la autoestima.

Por otro lado, González-Oya (2004) distingue cuatro niveles de actuación en relación con la autoconfianza:
1. Falta de confianza: deportistas que teniendo recursos, adolecen de la confianza necesaria para emplearlos frente a situaciones bajo presión ambiental, por ejemplo, cuando en un deporte se va perdiendo.

2. Exceso de confianza: se observa en aquellos deportistas que creen que no tienen que prepararse porque consideran que tienen sobrada capacidad para realizar la tarea, se creen los mejores.

3. Falsa confianza: se caracteriza por tener una actitud hacia los demás que no refleja la desconfianza y preocupación que experimenta el deportista en su interior.

4. Confianza óptima: es la que se pretende conseguir, según la cual es preciso que crean que van a rendir al máximo nivel de preparación, a partir de establecer una serie de objetivos realistas a su alcance.

El entrenador es una figura clave para ayudar al joven deportista a alcanzar su nivel óptimo de autoconfianza, ya que influye notablemente en su preparación, actuación, formación y funcionamiento general. Si el deportista confía en la eficacia de su entrenador, es muy probable que, indirectamente, se considere él mismo más eficaz, pues la eficacia del entrenador será un importante recurso en el que se podrá apoyar. Eso ocurrirá a condición que se fortalezca a la vez la autoconfianza con independencia del entrenador, pues una dependencia exclusiva o muy acusada sobre su persona tarde o temprano resultará perjudicial (Buceta, 1996). Asimismo, según Buceta (2014), la confianza en el entrenador puede desarrollarse a través de distintos elementos, como: la transmisión de sus conocimientos; su experiencia; su prestigio; su imagen pública y su credibilidad.

Del mismo modo, el diseño de programas de intervención por parte de los entrenadores/educadores que incluyan actividades físico-deportivas novedosas, de aventura o poco conocidas por los alumnos/as, formuladas a modo de reto, que incluyan diferentes niveles de dificultad, dan la posibilidad a todos de hacerlo bien y mejorar su autoconfianza, llegando a ser físicamente más activos al participar en estas actividades (Baena \& Granero, 2013; Nichols \& Fines, 1995). Por tanto, las actividades deberían estructurarse de manera que el éxito y el dominio 
sean posibles, pero sobre todo probables (Kimball \& Bacon, 1993).

Además, el empleo de algunas estrategias los días previos a la competición, tales como autoinstrucciones, visualización, o estrategias de afrontamiento del estrés, pueden ayudar a los jóvenes deportistas a mejorar su autoconfianza (Bertollo, Saltarelli, \& Robazza, 2009; Gould, Eklund, \& Jackson, 1993; Hatzigeorgiadis, Zourbanos, Mpoumpaki, \& Theodorakis, 2009; Mamassis \& Dogamis, 2004). Por ejemplo, los días previos a la competición, los técnicos podrían centrarse en reforzar las acciones que el deportista hace bien, destacando los aspectos positivos de ejecución motriz o acciones favorecedoras de un estado emocional positivo, sin incidir en aquellas técnicas o secuencias tácticas que el deportista aún no domina. Otra estrategia podría consistir en hacer entrenamientos en los que el deportista acumule muchas proyecciones y acciones exitosas, por las características de la tarea, o del rival. En esa línea, podría ser muy adecuada la visualización de filmaciones sobre competiciones anteriores en las que el deportista sale airoso, de forma que se vea capaz de repetirlo porque ya lo hizo bien antes. Además, realizar un estudio de rivales y trabajarlo con la antelación suficiente puede hacer que se rebaje el grado de incertidumbre, así como los niveles de ansiedad cognitiva y somática, aumentándose la autoconfianza. Todas estas consideraciones podrían ser beneficiosas en el fomento de la autoconfianza en el deporte en edad escolar, con las conocidas ventajas que esto conlleva al mediatizar las relaciones entre ansiedad $\mathrm{y}$ rendimiento (López-Torres, Torregrosa, \& Roca, 2007).

En suma, la autoconfianza es uno de los mejores pronósticos de éxito competitivo, ya que está íntimamente relacionada con la ejecución y con las estrategias que utiliza el deportista para resolver las diferentes situaciones de competición (Gómez-López, Granero, \& Isorna, 2013).

El objetivo de este estudio es analizar el estado de autoconfianza en el deporte en edad escolar que realizan los alumnos/as de entre $12 \mathrm{y}$ 18 años en Castilla-La Mancha, en función de su género, tipo de deporte y edad.

\section{Método}

La investigación siguió una metodología cuantitativa de corte descriptivo (Alvira, 2002). En el desarrollo de la metodología, los procedimientos que se siguieron fueron los propios de la encuesta, ya que se realizó la aplicación de un procedimiento estandarizado de recogida de la información mediante preguntas dirigidas a la muestra de la población estudiada (Lussier \& Kimball, 2008).

\section{Selección de la población y muestra}

Para estimar el universo general del estudio se utilizaron listados indirectos, que posibilitan la utilización de unidades de la muestra, debido a la inexistencia de un censo específico de personas entre 12 y 18 años que practiquen deporte escolar en algún club y/o escuela deportiva en Castilla-La Mancha. Tal y como expone Martínez del Castillo (1991), es necesario la utilización de listados indirectos para establecer el universo de trabajo para dicha población de estudios ya que, la identificación y localización de las unidades de las que se obtiene la información es muy compleja y difícil. Los listados utilizados en esta investigación fueron los siguientes:

1. Listado según edad y género en cada una de las cinco provincias de la Comunidad Autónoma de Castilla-La Mancha (Instituto Nacional de Estadística, 1 de julio de 2013).

2. Listado de municipios y población de la Comunidad Autónoma de Castilla-La Mancha (Instituto Nacional de Estadística, 1 de enero de 2012).

A partir de estos listados y de los valores obtenidos, se sumaron, aproximadamente, 147.166 personas que constituyen el total de la población objeto de estudio.

La muestra registró las siguientes características: Se incluyeron 816 deportistas (50.3\% varones y $49.7 \%$ mujeres) entre 12 y 18 años $(33.3 \%$ de 12 y 13 años, $33.3 \%$ de 14 y 15 años y $33.3 \%$ de 16 a 18 años) pertenecientes a clubes y/o escuelas deportivas de Castilla-La Mancha. Para el cálculo del tamaño de la muestra se consideraron varios aspectos: la población era infinita; se recurrió en la varianza poblacional al supuesto más desfavorable donde "P" y "Q" eran 
iguales con el 50\% cada uno; el intervalo de confianza era del $95.5 \%$, con un margen de error $\mathrm{de} \pm 3.55 \%$.

La afijación de la muestra fue proporcional a la distribución de las personas según tamaño demográfico de los municipios y según la situación geográfica de las diferentes provincias de Castilla-La Mancha. Además, se realizaron diversos sub-muestreos para dispersar la muestra y que influyese de forma positiva en la precisión de las estimaciones (Rodríguez Osuna, 2002).

Asimismo, se decidió entrevistar como máximo a dos equipos objeto de estudio en cada club y/o escuela deportiva. Al realizar este procedimiento se dispersó la muestra e influyó positivamente en la precisión de las estimaciones, disminuyéndose además el error muestral (Cea D’Ancona, 2001; Rodríguez Osuna, 1991).

El tipo de muestreo utilizado fue el muestreo aleatorio por conglomerados, dentro del cual se utilizó un muestreo polietápico, estratificado en primera fase por conglomerados, ya que se siguió una secuencia de etapas (aplicándose un muestreo aleatorio en cada una de las etapas) de selección de unidades muestrales (conglomerados) de mayor a menor rango, hasta llegar a los individuos constituyentes de la muestra (Arnal, Del Rincón, \& Latorre, 1992; Cea D’Ancona, 2001; Rodríguez Osuna, 2002).

\section{Instrumento}

El instrumento utilizado en el estudio fue el cuestionario Características Psicológicas relacionadas con el Rendimiento Deportivo (CPRD) de Gimeno, Buceta, y Pérez-Llantada (1994). Estos autores lo crearon con el fin de disponer de una herramienta similar en el contexto de deportistas españoles, al cuestionario Psychological Skills Inventory for Sports (PSIS) de Mahoney, Gabriel, y Perkins (1987). El CPRD presenta una alta consistencia interna en el conjunto de los 55 ítems que lo componen $(\alpha=.85)$, y valores superiores a .70 en tres de las cinco dimensiones que definen su estructura factorial: control del estrés $(\alpha=.88)$, influencia de la evaluación del rendimiento - que es una variable de estrés específica $-(\alpha=.72)$, motivación $(\alpha=.67)$, habilidad mental $(\alpha=.34)$ y cohesión de equipo $(\alpha=.78)$. El cuestionario CPRD conserva el formato de respuesta tipo Likert de cinco puntos Revista Iberoamericana de Diagnóstico y Evaluación - e Avaliação Psicológica. RIDEP · No44 · Vol.2 · 158-171 · 2017 del cuestionario PSIS, pero con la inclusión de una opción de respuesta adicional para aquellos casos en los que el deportista «no entiende el ítem», con el fin de evitar respuestas «en blanco» o en la posición central. Los ítems incluyen contenidos relacionados con las principales variables psicológicas relacionadas con el rendimiento, constituyendo una herramienta útil para fortalecer los elementos psicológicos en la formación deportiva. Consta de preguntas cerradas y categorizadas, donde el orden de las mismas es inflexible. Además, algunas de ellas son de control (para comprobar la congruencia y sinceridad de las respuestas del entrevistado) y otras de batería (conjunto de preguntas sobre la misma cuestión que se complementan). Para el adecuado desarrollo del presente trabajo, se seleccionó la dimensión control del estrés $(\alpha=$ .88), analizándose en la misma los ítems correspondientes a autoconfianza.

\section{Procedimiento}

Tras obtener la aprobación de colaboración por parte de los entrenadores, y/o presidentes de los clubes y escuelas deportivas participantes en el estudio, así como el consentimiento informado por escrito de los padres de los jóvenes deportistas, los cuestionarios fueron respondidos por los jóvenes deportistas de forma colectiva y anónima en la instalación deportiva. Los investigadores estuvieron presentes durante la administración de las pruebas para aclarar posibles dudas y verificar la cumplimentación independiente por parte de los participantes.

\section{Análisis estadístico}

Para realizar el análisis estadístico se recurrió al programa SPSS v.20.0 (SPSS Inc., EE.UU). Se utilizaron pruebas de estadística descriptiva e inferencial. En la estadística descriptiva, se realizó un análisis bivariado mediante tablas de frecuencia relativas (porcentajes). Además, se hallaron datos estadísticos de frecuencia tales como: media y desviaciones típicas.

En la estadística inferencial, para comprobar la normalidad de las distribuciones, se utilizó el test de Kolmogorov-Smirnov. En las variables que cumplían una distribución normal, como prueba inferencial se utilizó la prueba $t$ de student para 
muestras independientes y ANOVA de un factor con el fin de analizar la relación existente entre las variables. Como prueba Post hoc se seleccionó la prueba de Tukey, usando el criterio estadístico de significación de $p<.05$.

Asimismo, se utilizó el test de Levene para evaluar la homogeneidad de las varianzas. Cuando había diferencia de varianzas en el test de Levene (<.05), se utilizó la prueba de Welch, con el fin de analizar la relación existente entre las variables.

\section{Resultados}

En la Tabla 1 se muestra el nivel de autoconfianza en relación con el género. Para ello, se llevó a cabo un estadístico de grupo con los ítems del cuestionario que miden la autoconfianza. En un primer momento, se hallaron diferencias de Medias en los cuatro ítems analizados, apreciándose en todos ellos mayores puntuaciones en los hombres. Una vez explorado y asumido el cumplimiento del criterio de Normalidad de Kolmogorov-Smirnov para muestras grandes (> 100 individuos), se procedió a realizar la prueba de Levene o prueba de homogeneidad de varianzas, en el que se apreciaron diferencias significativas entre varianzas en tres de los ítems analizados, el primero $(F=8.09, p=.005)$; el cuarto y el último $(F=26.38, p=.00)$. Por último, se procedió a realizar la Prueba $T$ para muestras independientes, que mostró como en todos los ítems analizados no se asumieron varianzas iguales: $t(813.23)=3.52$, $p=.00 ; t(809.47)=-2.56, p=.01 ; t(803.20)=-2.94$, $p=.003 ; t(769.81)=5.17, p=.00$, respectivamente. Por tanto, existen diferencias significativas en las respuestas entre ambos géneros, siendo los hombres que practican deportes extraescolares, los que manifiestan una mayor autoconfianza.

Respecto a la autoconfianza, en relación con la modalidad deportiva, en un primer momento, en la prueba ANOVA de un factor se apreciaron diferencias de Medias entre los diferentes deportes. En la prueba de homogeneidad de varianzas de Levene, en el primer ítem analizado (Tengo una gran confianza en mi técnica) existía diferencia de varianzas, $F(9,806)=2.19, p=.02$, al igual que en el segundo ítem analizado (No se necesita mucho para que se debilite mi confianza en mí mismo), $F(9,806)=3.11, p=.001$, por lo que se consultó la prueba de Welch. Esta prueba mostró, que en el primer ítem analizado (ver más arriba), $\quad F \quad(9,328.30)=2.54, \quad p<.05, \quad$ había diferencias significativas entre al menos dos deportes. Para verificar en qué deportes las diferencias eran estadísticamente significativas, se halló la prueba Post hoc de Tukey. Esta prueba mostró la existencia de diferencias estadísticamente significativas entre los deportistas que practican Fútbol y Judo, que manifiestan tener una gran confianza en su técnica, con respecto a deportistas que practican Voleibol, que manifiestan tener una menor confianza en su técnica (Tabla 2).

Además, en relación con el tipo de deporte (individual-colectivo), se llevó a cabo un estadístico de grupo con los ítems del cuestionario que miden la autoconfianza. En un primer momento, se hallaron diferencias de Medias en el segundo de los ítems analizados. Se procedió a realizar la prueba de Levene o prueba de homogeneidad de varianzas, en el que se apreciaron diferencias significativas entre varianzas en el segundo de los ítems analizados $(\mathrm{F}=4.13, p=.04)$. Por último, se procedió a realizar la Prueba $T$ para muestras independientes, que mostró como nuevamente en el segundo de los ítems analizados (No se necesita mucho para que se debilite mi confianza en mí mismo) no se asumieron varianzas iguales, $t(810.62)=-2.32$, $p=.02$. Por tanto, existen diferencias significativas en las respuestas de autoconfianza entre ambos tipos de deporte, siendo en los deportes individuales donde se debilita menos la autoconfianza de los deportistas. Por consiguiente, existen mayores niveles de autoconfianza en deportistas que practican deportes individuales (Tabla 3).

Por último, respecto a la autoconfianza en relación con la edad, en un primer momento, no se apreciaron diferencias de Medias en ninguno de los ítems analizados. A continuación, en la prueba de homogeneidad de varianzas de Levene, existía igualdad de varianzas en todos los ítems analizados. Posteriormente, se procedió a consultar el ANOVA, confirmándose la igualdad de varianzas en todos los ítems analizados, $F$ $(2,813)=.41, \quad p>.05 ; \quad F \quad(2,813)=3.13, \quad p>.05 ;$ $F(2,813)=1.43, p>.05 ; F(2,813)=1.48, p>.05$, 
Tabla 1. Autoconfianza en función del género

\begin{tabular}{lcccccc}
\hline Ítems autoconfianza & Género & $n$ & $M$ & $D T$ & $t$ & Sig. \\
\hline $\begin{array}{l}\text { Tengo una gran confianza } \\
\text { en mi técnica }\end{array}$ & $\begin{array}{l}\text { Masculino } \\
\text { Femenino }\end{array}$ & 411 & 3.49 & 1.10 & 3.52 & $.00^{*}$ \\
$\begin{array}{l}\text { No se necesita mucho } \\
\text { para que se debilite mi } \\
\text { confianza en mí mismo }\end{array}$ & Masculino & 411 & 2.19 & 1.16 & -2.56 & $.01^{*}$ \\
& Femenino & 405 & 2.41 & 1.23 & -2.56 & $.01^{*}$ \\
$\begin{array}{l}\text { Mi confianza en mí } \\
\text { mismo/a es muy inestable }\end{array}$ & Masculino & 411 & 2.29 & 1.23 & -2.94 & $.003^{*}$ \\
& Femenino & 405 & 2.56 & 1.36 & -2.94 & $.003^{*}$ \\
$\begin{array}{l}\text { Tengo fe en mí mismo/a } \\
\text { Masculino }\end{array}$ & 411 & 4.17 & .92 & 5.17 & $.00^{*}$ \\
\hline${ }^{*}<.05$ & Femenino & 405 & 3.79 & 1.16 & 5.17 & $.00^{*}$ \\
\hline
\end{tabular}

Tabla 2. Autoconfianza en función de la modalidad deportiva (Análisis ítem "Tengo una gran confianza en mi técnica”), (HSD de Tukey)

\begin{tabular}{lccc}
\hline \multirow{2}{*}{ Deporte } & $n$ & \multicolumn{2}{c}{$\begin{array}{c}\text { Subconjunto } \\
\text { para alfa }=.05\end{array}$} \\
\cline { 2 - 4 } & 1 & 1 & 2 \\
\hline Voleibol & 81 & 3.04 & \\
Baloncesto & 82 & 3.12 & 3.12 \\
G. Rítmica & 81 & 3.26 & 3.26 \\
Natación & 82 & 3.27 & 3.27 \\
Fútbol Sala & 82 & 3.28 & 3.28 \\
Tenis & 82 & 3.39 & 3.39 \\
Atletismo & 81 & 3.48 & 3.48 \\
Balonmano & 82 & 3.50 & 3.50 \\
Judo & 81 & & 3.59 \\
Fútbol & 82 & & 3.65 \\
Sig. & & .18 & .07 \\
\hline
\end{tabular}

respectivamente. Por tanto, no existen diferencias estadísticamente significativas en la variable autoconfianza en relación con la edad.

\section{Discusión}

Los resultados con referencia al género confirman que existen diferencias estadísticamente significativas, siendo los hombres los que presentan una autoconfianza mayor. Estos resultados coinciden con otros estudios previos realizados en el deporte en edad escolar, que también mostraron mayores niveles de autoconfianza en los hombres con respecto a las mujeres (Abrahamsen, Roberts, \& Pensgaard, 2008; Balaguer, Escartí, Soler, \& Jiménez, 1990; Brunet \& Sabiston, 2008; Carazo \& Araya, 2010; Goñi, Ruiz de Azúa, \& Rodríguez, 2006; Klomsten, Marsh, \& Skaalvik, 2005; LeónPrados, Fuentes, \& Calvo, 2011; Lesser \& Murphy, 1988; Martens, Vealey, \& Burton, 1990; Meyers, Bourgeois, LeUnes, \& Murray, 1999; Moraes, 1987; Moreno, Moreno, \& Cervelló, 2013; Rokka, Mavridis, Bebetsos, \& Mavridis, 2009; Sagar \& Jowett, 2012; Sánchez-Alcaraz \& Gómez, 2014; Scanlan \& Passer, 1979; Sewell \& Edmondson, 1996; Wark \& Witting, 1979).

Esta tendencia podría atribuirse a que los hombres tienen mayores oportunidades en el universo competitivo, hecho que les aportaría mayores niveles de práctica, de experiencia y de adherencia deportiva y, por tanto, mayores niveles de autoconfianza (Contreras, Fernández, García, Palou, \& Ponseti, 2010; Gómez-Mármol \& De la Cruz, 2013; Jones \& Cale, 1989). Asimismo, las mujeres muestran más preocupaciones ante los errores cometidos en las competiciones y un mayor miedo al fracaso en las mismas, lo que sin duda, debilita su autoconfianza (Frost \& Henderson, 1991). Estas experiencias negativas 
Tabla 3. Autoconfianza en función del tipo de deporte

\begin{tabular}{lcccccc}
\hline Ítems autoconfianza & $\begin{array}{c}\text { Tipo de } \\
\text { Deporte }\end{array}$ & $\mathrm{n}$ & $\mathrm{M}$ & $\mathrm{DT}$ & $t$ & Sig. \\
\hline $\begin{array}{l}\text { Tengo una gran confianza } \\
\text { en mi técnica }\end{array}$ & Individual & 407 & 3.40 & 1.08 & 1.02 & .30 \\
& Colectivo & 409 & 3.32 & 1.14 & 1.02 & .30 \\
$\begin{array}{l}\text { No se necesita mucho } \\
\text { para que se debilite mi } \\
\text { confianza en mí mismo }\end{array}$ & Individual & 407 & 2.20 & 1.15 & -2.32 & $.02^{*}$ \\
& Colectivo & 409 & 2.40 & 1.23 & -2.32 & $.02^{*}$ \\
$\begin{array}{l}\text { Mi confianza en mí } \\
\text { mismo/a es muy inestable }\end{array}$ & Individual & 407 & 2.34 & 1.27 & -1.77 & .07 \\
& Colectivo & 409 & 2.50 & 1.33 & -1.77 & .07 \\
Tengo fe en mí mismo/a & Individual & 407 & 3.97 & 1.06 & -.32 & .74 \\
& Colectivo & 409 & 3.99 & 1.08 & -.32 & .74 \\
\hline$p .05$ & & & & & &
\end{tabular}

podrían propiciar el abandono prematuro de su actividad física (Knowles, Niven, \& Fawkner, 2013; Slater \& Tiggemann, 2010). Además, se relacionan bajos niveles de ansiedad competitiva en los hombres con altos niveles de autoconfianza, $\mathrm{y}$ altos niveles de ansiedad competitiva en las mujeres con bajos niveles de autoconfianza (Harter, 1999; Navlet, 2012). También, otra de las posibles razones puede residir en que las mujeres jóvenes perciben que sus cuerpos son mirados, comentados y evaluados por otros debido a los cambios físicos puberales que experimentan, pudiendo contribuir a una disminución en la autoconfianza respecto de la actividad deportiva desarrollada (Linder, Grabe, \& Hyde, 2007). Por tanto, la gran mayoría de estudios indican la existencia de diferencias de género en los niveles de autoconfianza durante la práctica deportiva. Es posible identificar a los factores biológicos y a las diferencias de roles, como principales responsables del mayor nivel de autoconfianza que manifiestan los hombres (Kling, Hyde, Showers, \& Buswell, 1999; Lewinsohn, Gotlib, Lewinsohn, Seeley, \& Allen, 1998; Matud, 2004; Montgomery \& Morris, 1994; O`Brien, Hanton, \& Mellalieu, 2003). No obstante, otros estudios afirmaron que no existían diferencias estadísticamente significativas de género en la variable autoconfianza (Martín, 2003; Montero, Moreno-Murcia, González Ponce, Pulido, \&
Cervelló, 2012; Munroe, Hall, \& Fishburne, 2008; Perry \& Williams, 1998; Pozo, 2007; Woo, 1996). Con referencia a la autoconfianza y la modalidad deportiva los resultados encontrados en este estudio confirman que existen diferencias estadísticamente significativas entre jóvenes deportistas practicantes de modalidades deportivas como Fútbol y Judo (mayor confianza en su técnica) con respecto a otras modalidades deportivas como Voleibol (menor confianza en su técnica). Estos resultados coinciden con otros estudios previos realizados en el deporte en edad escolar, en los que también existieron diferencias significativas entre diversas modalidades deportivas en relación con la autoconfianza (Buceta, López de la Llave, Pérez-Llantada, Vallejo, \& Del Pino, 2003; Burke \& Jin, 1996; Esnaola, 2005; Jaenes \& Caracuel, 2005; Jaenes, Peñaloza, Navarrete, \& Bohórquez, 2012). En este sentido, Jaenes et al. (2012) relacionan los deportes de resistencia practicados por los jóvenes deportistas con mayores niveles de autoconfianza, argumentando como posibles causas que al tratarse de deportistas populares, solamente existe un compromiso personal de participación, a los que no se les presupone una orientación sobre el resultado. Además, otra posible explicación radica en la adherencia a la actividad, siendo posible hipotetizar, que sólo se mantienen compitiendo aquellos cuyos niveles de ansiedad son bajos y altos los de autoconfianza. Esnaola (2005) alerta 
que, por el contrario, determinados deportes estéticos, centrados única y exclusivamente en el culto hacia el cuerpo, en la imagen corporal, en la preocupación excesiva por el peso, pueden generar alteraciones en la percepción del autoconcepto físico, disminuyendo los niveles de autoconfianza de los jóvenes deportistas.

En cuanto a la autoconfianza y al tipo de deporte (individual-colectivo), los resultados de este estudio también confirman la existencia de diferencias estadísticamente significativas. Los deportistas que practican deportes individuales muestran mayores niveles de autoconfianza con respecto a los deportistas que practican deportes colectivos. Estos hallazgos coinciden con otros estudios previos realizados en el deporte en edad escolar (Radzi, Yusof, \& Zakaria, 2013; Sagar \& Jowett, 2012). Algunas de las posibles causas de los mismos se pueden deber a que en los deportes de equipo los fallos y actuaciones pobres de los jóvenes deportistas afectan a sus compañeros de equipo y a los resultados de la competición. Esto conlleva a que se juzgue continuamente y se evalúe cada una de las acciones por parte del equipo, generándose en ocasiones una excesiva presión en el joven deportista para intentar evitar errores que perjudiquen al equipo. Esta situación se traduce en una mayor ansiedad y pérdida de niveles de autoconfianza (Sagar \& Jowett, 2012). Sin embargo, otros estudios mostraron mayores niveles de autoconfianza en deportistas que practicaban deportes colectivos (Martens et al., 1990; Zeng, 2003).

Por otra parte, numerosos estudios indican, que los jóvenes deportistas que practican algún tipo de deporte, con respecto a los que no lo practican, muestran mayores niveles de autoconfianza. Además, en el caso de los jóvenes deportistas que practican algún tipo de deporte, los que tienen mayor experiencia y práctica deportiva, también muestran mayores niveles de autoconfianza (Dieppa, Machargo, Luján, \& Guillén, 2009; Goñi, Ruiz de Azúa, \& Rodríguez, 2005; Hanton, Neil, Mellalieu, \& Fletcher, 2008; Leith, 1994; Mellalieu, Neil, \& Hanton, 2006; Molero, Ortega, Caliente, \& Zagalaz, 2010; Parfitt \& Pates, 1999; Pozo, 2007; Reche, Cepero, \& Rojas, 2010; Rokka et al., 2009; Sánchez-Alcaraz \& Gómez, 2014; Wilson \& Raglin, 1997; Woodman \& Hardy, 2003). Sin embargo, en otros Revista Iberoamericana de Diagnóstico y Evaluación - e Avaliação Psicológica. RIDEP · No44 · Vol.2 · 158-171 2017 estudios no aparecieron diferencias estadísticamente significativas en este sentido (Godoy, Vélez, \& Pradas, 2007; Munroe et al., 2008).

Con referencia a la autoconfianza y la edad los resultados encontrados confirman que no existen diferencias estadísticamente significativas entre jóvenes deportistas pertenecientes a diversas categorías en el deporte en la edad escolar. Estos resultados coinciden con otros estudios realizados previamente (Munroe et al., 2008; Soltani et al., 2012), en el que no se hallaron diferencias significativas entre jóvenes deportistas de competición-élite y de recreación-no élite, hallándose, además, altos niveles de autoconfianza en ambos estudios. Igualmente, en otros estudios similares (Hernández, Olmedilla, \& Ortega 2008; Moreno, Claver, Gil, Moreno, \& Jiménez, 2014; Tsopani, Dallas, \& Skordilis, 2011), tampoco se hallaron diferencias estadísticamente significativas entre deportistas de 12 y 13 años con respecto a deportistas de 14 y 15 años. Uno de los motivos por el que no aparecen diferencias significativas en los niveles de autoconfianza en jóvenes deportistas de diferentes edades, quizá pueda deberse a que antes de la competición perciban que están suficientemente preparados para afrontarla, que es asequible a sus posibilidades (aunque luego en la práctica no sea realmente así) y por tanto la afronten con altos niveles de autoconfianza (Hernández et al., 2008). Otra posible razón sería que los deportistas de diferentes edades entienden no solo como muy importante el entrenamiento mental para su rendimiento, sino además, que todas las habilidades importantes para rendir hay que entrenarlas en condiciones de concentración y pertinencia (Cepeda, Montero, García-González, León, \& Iglesias, 2011). Complementariamente, los años de edad o de práctica no siempre son sinónimos de una mayor experiencia (Hanton, Cropley, \& Lee, 2009).

Sin embargo, otros estudios indican que existen diferencias estadísticamente significativas. En ellos en los deportistas de mayor edad aparecen mayores niveles de autoconfianza (Bebetsos, Zouboulias, Antoniou, \& Kourtesis, 2013; Hernández et al., 2008; Rokka et al., 2009; Sagar \& Jowett, 2012). Posiblemente esto pueda deberse a una consecuencia lógica adaptativa, 
ligada a una mayor experiencia en situaciones competitivas estresantes, junto con un mayor dominio técnico-táctico adquirido y en algunos casos, con objetivos de rendimiento muy marcados, lo que pueda explicar la mayor percepción de autoconfianza en los deportistas de mayor edad (Bebetsos et al., 2013; Hernández et al., 2008). Por el contrario, otros estudios indican que los jóvenes deportistas de menor edad muestran mayores niveles de autoconfianza (Guillén \& Álvarez-Malé, 2010). Es posible que esto se deba a que los deportistas de mayor edad comienzan a tener metas más competitivas y se encuentran en contextos deportivos más inciertos, con la consecuente aparición simultánea de síntomas de ansiedad y estrés, lo que repercute en un menor nivel de autoconfianza (Conroy, 2004).

Finalmente, en los hallazgos en los que convergen las principales investigaciones refieren a que a mayor nivel competitivo del deportista, mayores son los niveles de autoconfianza encontrados (Kim, Chung, Park, \& Shin, 2009; León-Prados et al., 2011; Lundqvist, Kenttä, \& Raglin, 2011; Montero et al., 2012; Pozo, 2007; Reche, Cepero, \& Rojas, 2013; Robazza, Pellizzari, Bertollo, \& Hanin, 2008; Wadey \& Hanton, 2007). Probablemente, los deportistas de mayor nivel deportivo tengan más experiencia en las situaciones competitivas, lo que hace que sepan interpretar mejor su propia competencia sobre el reto al que van a enfrentar. Al respecto debe tenerse en cuenta que la autoconfianza es una evaluación cognitiva de las propias capacidades para afrontar un desafío y hace referencia a una evaluación subjetiva de la competencia (Montero et al., 2012).

\section{Conclusiones}

A la vista de la discusión, se puede afirmar que los resultados del estudio coinciden con otros estudios realizados previamente $\mathrm{y}$ resultan contradictorios con otros, motivo por el cual no son concluyentes, por lo que deben asumirse con cierta cautela.

Sin embargo, estos resultados pueden tener un sentido práctico, tanto para el entrenamiento como para la competición, ya que permiten proporcionar una información mayor y mejor a los entrenadores de los jóvenes deportistas. Información sobre las emociones que están frecuentemente relacionadas con desafíos competitivos y permiten ayudar a trabajar habilidades y estrategias de afrontamiento para que el deportista logre tener una mejor predisposición a la hora de afrontar la competición, mejorando su autoconfianza, lo que conducirá probablemente a tener un mejor rendimiento.

Una de las principales limitaciones del estudio se refiere a la "falsa confianza" que pueden manifestar los jóvenes deportistas, teniendo en cuenta la "rigidez-flexibilidad" en las respuestas. Así, la puntuación máxima obtenida por las respuestas de un deportista a todos los ítems podría indicar que tiene una autoconfianza muy fuerte, pero también que quizá esté enmascarando déficits de autoconfianza. Esta duda deberá despejarse, en la medida posible, a través de la observación continuada y la entrevista personal. Se debe considerar su disposición, o rechazo a aceptar, analizar y discutir sus "puntos débiles", su capacidad para admitir las críticas, su tendencia a atribuir el fracaso a causas externas, su permanente "huída hacia delante" y otros elementos significativos.

Como futura línea de investigación, se podría realizar un seguimiento longitudinal que devele información suficiente para conocer en mayor profundidad cómo los deportistas de base perciben la autoconfianza en diferentes momentos y situaciones de la temporada. De este modo resultaría posible comprobar si resulta adecuado o no aplicar estrategias psicológicas a edades tempranas y, en tal caso, determinar el momento idóneo para su puesta en marcha.

No obstante, una medida imprescindible, es la adopción por parte de los entrenadores, padres y jóvenes deportistas, de medidas de prevención de control del estrés y ansiedad, a fin de reducir sus niveles en la práctica deportiva en la edad escolar, potenciando paralelamente su autoconfianza.

\section{Referencias}

Abrahamsen, F. E., Roberts, G. C., \& Pensgaard, A. M. (2008). Achievement goals and gender effects on multidimensional anxiety in 
national elite sport. Psychology of Sport and Exercise, 9(4), 449-464.

Alvira, F. (2002). Metodología de la evaluación de programas: Un enfoque práctico. Madrid: CIS. Cuadernos Metodológicos.

Arnal, J., Del Rincón, D., \& Latorre, A. (1992). Investigación educativa. Fundamentos y metodología. Barcelona: Labor.

Baena, A., \& Granero, A. (2013). Efecto de un programa de educación de aventura en la orientación al aprendizaje, satisfacción y autoconcepto en secundaria. Revista Iberoamericana de Diagnóstico y Evaluación - e Avaliação Psicológica, 36(2), 163-182.

Balaguer, I., Escartí, A., Soler, M. J., \& Jiménez, C. (1990, abril). Influencia de la autoconfianza en el deporte y de la orientación competitiva sobre la ejecución en un grupo de nadadores orientados a la competición: II Congreso del Colegio Oficial de Psicólogos, 90-95, Valencia, Colegio Oficial de Psicólogos de Madrid.

Bandura, A. (1977). Social learning theory. New York: General Learning Press.

Bebetsos, E., Zouboulias, S., Antoniou, P., \& Kourtesis, T. (2013). Do anxiety, anger and aggression differentiate elite water-polo players? Journal of Physical Education and Sport, 13(2), 209-212.

Bertollo, M., Saltarelli, B., \& Robazza, C. (2009). Mental preparation strategies of elite modern pentathletes. Psychology of Sport and Exercise, 10, 244-254.

Brunet, J., \& Sabiston, C. M. (2008). A selfdetermination theory approach to understanding the relationship between social physique anxiety and physical activity. Abstract published in Journal of Sport \& Exercise Psychology, 30, 156-157.

Buceta, J. M. (1996). Psicología y lesiones deportivas: Prevención y recuperación. Madrid: Dykinson.

Buceta, J. M. (1999). Psicología del alto rendimiento deportivo. Madrid: Real Federación Española de Fútbol.

Buceta, J. M. (2004). Estrategias psicológicas para entrenadores de deportistas jóvenes. Madrid: Dykinson.

Buceta, J. M. (2014, octubre). Intervención psicológica en deportes de equipo.
Comunicación presentada en la Conferencia correspondiente a las XVII Jornadas de Actualización en Psicología del Deporte. Madrid: UNED.

Buceta, J. M., López de la Llave, A., PérezLlantada, M. C., Vallejo, M., \& Del Pino, M. D. (2003). Estado psicológico de los corredores populares de maratón en los días anteriores a la prueba. Psicothema, 15(2), 273-277.

Burke, S. T., \& Jin, P. (1996). "Predicting performance from a triathlon event / Predire la performance d'une competition de triathlon". Journal of Sport Behavior, 19(4), 272-287.

Carazo, P., \& Araya, G. (2010). Perfil de rasgos psicológicos para el rendimiento deportivo en hombres y mujeres practicantes de taekwondo. Revista Iberoamericana de Psicología del Ejercicio y el Deporte, 5, 253266.

Cea D’Ancona, M. A. (2001). Metodología cuantitativa. Estrategias y técnicas de investigación social. Madrid: Síntesis.

Cepeda, M., Montero, C., García-González, L., León, B., \& Iglesias, D. (2011). La percepción de judocas de distinto nivel de pericia respecto a las actividades de entrenamiento específicas que contribuyen al rendimiento. Archivos de Medicina del Deporte, 145, 331340.

Conroy, D. E. (2004). The unique psychological meanings of multidimensional fears of failing. Journal of Sports \& Exercise Psychology, 26, 484-491.

Contreras, O., Fernández, J., García, L. M., Palou, P., \& Ponseti, J. (2010). El autoconcepto físico y su relación con la práctica deportiva en estudiantes adolescentes. Revista de Psicología del Deporte, 19(1), 23-39.

Cruz, J. (1997). Factores motivacionales en el deporte infantil y asesoramiento psicológico a entrenadores y padres. En J. Cruz (Ed.), Psicología del deporte (pp. 147-176). Madrid: Síntesis.

Dieppa, M., Machargo, J., Luján, I., \& Guillén, F. (2009). Autoconcepto general y físico en jóvenes españoles y brasileños que practican actividad física versus no practicantes. Revista de Psicología del Deporte, 17(2), 221-239. 
Esnaola, I. (2005). Autoconcepto físico y satisfacción corporal en mujeres adolescentes según el tipo de deporte practicado. Apunts. Educación Física y Deportes, 2, 5-12.

Frost, R. O., \& Henderson, K. J. (1991). Perfectionism and reactions to athletic competition. Journal of Sport \& Exercise Psychology, 13, 323-335.

Gimeno, F., Buceta, J. M., \& Pérez-Llantada, M. C. (1994). El cuestionario de Características Psicológicas relacionadas con el Rendimiento Deportivo (CPRD): Características psicométricas. En A. López de la Llave, M. C. Pérez-Llantada, \& J. M. Buceta (Eds.), Investigaciones breves en psicología del deporte (pp. 65-76). Madrid: Dykinson.

Godoy, D., Vélez, M., \& Pradas, F. (2007). Nivel de dominio de las habilidades psicológicas en jóvenes jugadores de deportes de raqueta y pala: Tenis de mesa y bádminton. Cuadernos de Psicología del Deporte, 7(1), 45-59.

Gómez-López, M., Granero, A., \& Isorna, M. (2013). Análisis de los factores psicológicos que afectan a los piragüistas en el alto rendimiento. Revista Iberoamericana de Diagnóstico y Evaluación - e Avaliação Psicológica, 35(1), 57-76.

Gómez-Mármol, A., \& De la Cruz, E. (2013). Gender and academic level differences on scholar physical education's perceived usefulness at secondary compulsory education. Journal of Sport \& Health Research, 5(2), 193-202.

González-Oya, J. L. (2004). Características psicológicas de árbitros de fútbol (Tesis doctoral inédita). Universidad de Vigo, Vigo (Pontevedra).

Goñi, A., Ruiz de Azúa, S., \& Rodríguez, A. (2005). La importancia concedida a diversos aspectos del yo físico: Un instrumento para su medida. Anales de Psicología, 21(1), 92-101.

Goñi, A., Ruiz de Azúa, S., \& Rodríguez, A. (2006). Cuestionario de Autoconcepto Físico $(C A F)$. Manual. Madrid: EOS.

Gould, D., Eklund, R. C., \& Jackson, S. A. (1993). Coping strategies used by U.S. Olympic wrestlers. Research Quarterly of Exercise \& Sport, 64, 83-93.

Guillén, F., \& Álvarez-Malé, M. L. (2010). Relación entre los motivos de la práctica deportiva y la ansiedad en jóvenes nadadores de competición. Revista Iberoamericana de Psicología del Ejercicio y el Deporte, 5(2), 233-251.

Hanton, S., Cropley, B., \& Lee, S. (2009). Reflective practice, experience, and the interpretation of anxiety symptoms. Journal of Sports Sciences, 27(5), 517- 533.

Hanton, S., Neil, R., Mellalieu, S. D., \& Fletcher, D. (2008). Competitive experience and performance status: An investigation into multidimensional anxiety and coping. European Journal of Sport Science, 8(3), 143152.

Harter, S. (1999). The construction of the self: A developmental perspective. New York: Guilford.

Hatzigeorgiadis, A., Zourbanos, N., Mpoumpaki, S., \& Theodorakis, Y. (2009). Mechanisms underlying the self-talk-performance relationship: The effects of self-talk on selfconfidence and anxiety. Psychology of Sport \& Exercise, 10, 186-192.

Hernández G., R., Olmedilla, A., \& Ortega, E. (2008). Ansiedad y autoconfianza de jóvenes judokas en situaciones competitivas de alta presión. Análise Psicológica, 4, 689-696.

INE (2012). Listado de municipios y población de la Comunidad Autónoma de Castilla-La Mancha (Instituto Nacional de Estadística, 1 de enero de 2012).

INE (2013). Listado según edad y género en cada una de las cinco provincias de la Comunidad Autónoma de Castilla-La Mancha (Instituto Nacional de Estadística, 1 de julio de 2013).

Jaenes, J. C., \& Caracuel, J. C. (2005). Maratón: Preparación psicológica para el entrenamiento y la competición. Córdoba: Almuzara.

Jaenes, J. C., Peñaloza, R., Navarrete, K. G., \& Bohórquez, M. R. (2012). Ansiedad y autoconfianza precompetitiva en triatletas. Revista Iberoamericana de Psicología del Ejercicio y el Deporte, 7(1), 113-124.

Jones, G., \& Cale, A. (1989). Relationships between multidimensional competitive anxiety and cognitive motor subcomponents of performance. Journal of Sports Sciences, 7 , 229-240. 
Kim, K. J., Chung, J. W., Park, S., \& Shin, J. T. (2009). Psychophysiological stress response during competition between elite and nonelite Korean junior golfers. International Journal of Sports Medicine, 30(7), 503-508.

Kimball, R. O., \& Bacon, S. B. (1993). The wilderness challenge model. En M. A. Gass (Ed.), Adventure therapy: Therapeutic applications of adventure programming (pp. 11-41). Dubuque, IA: Kendall/Hunt Publishing Company.

Kling, K. C., Hyde, J. S., Showers, C. J., \& Buswell, B. N. (1999). Gender differences in self-esteem: A metaanalysis. Psychological Bulletin, 125(4), 470-500.

Klomsten, A. T., Marsh, H. W., \& Skaalvik, E. M., (2005). 'Adolescents' perceptions of masculine and feminine values in sport and physical education: A study of gender differences'. Sex Roles, 52(9-10), 625-636.

Knowles, A. M., Niven, A., \& Fawkner, S. (2013). 'Once upon a time I used to be active'. Adopting a narrative approach to understanding physical activity behaviour in adolescent girls. Qualitative Research in Sport, Exercise \& Health, 6(1), 62-76. doi:10.1080/2 159676X.2013.766816

Leith, L. M. (1994). Foundations of exercise and mental health. Morgantown, WV: Fitness Information Technology.

León-Prados, J. A., Fuentes, I., \& Calvo, A. (2011). Ansiedad estado y autoconfianza precompetitiva en gimnastas. International Journal of Sport Science, 7(6), 76-91.

Lesser, M., \& Murphy, S. M. (1988, agosto). The Psychological Skills Inventory for Sports (PSIS): Normative and reliability data. Paper presented at the annual meeting of the American Psychological Association, Atlanta.

Lewinsohn, P. M., Gotlib, I. H., Lewinsohn, M., Seeley, J. R., \& Allen, N. B. (1998). Gender differences in anxiety disorders and anxiety symptoms in adolescents. Journal of Abnormal Psychology, 107(1), 109-117.

Linder, S. M., Grabe, S., \& Hyde, J. S. (2007). Gender, pubertal development, and peer sexual harassment predict objectified body consciousness in early adolescence. Journal of Research on Adolescence, 17(4), 723-742. doi:10.1111/j.15327795.2007.00544.x
López-Torres, M., Torregrosa, M., \& Roca, J. (2007). Características del «Flow», ansiedad y estado emocional en relación con el rendimiento de deportistas de elite. Cuadernos de Psicología del Deporte, 7(1), 25-44.

Lundqvist, C., Kenttä, G., \& Raglin, J. S. (2011). Directional anxiety responses in elite and subelite young athletes: Intensity of anxiety symptoms matters. Scandinavian Journal of Medicine \& Science in Sports, 21, 853-862.

Lussier, R. N., \& Kimball, D. C. (2008). Applied sport management skills. Champaign, IL: Human Kinetics.

Mahoney, M. J., Gabriel, T. J., \& Perkins, T. S. (1987). Psychological skills and exceptional athletic performance. The Sport Psychologist, 1, 181-199.

Mamassis, G., \& Dogamis, G. (2004). The effects of a mental training program on juniors precompetitive anxiety, self-confidence, and tennis performance. Journal of Applied of Sport Psychology, 16, 118-137.

Martens, R., Vealey, R. S., \& Burton, D. (1990). Competitive anxiety in sport. Champaign, IL: Human Kinetics.

Martín, G. (2003). La mujer futbolista desde la perspectiva psicológica. Cuadernos de Psicología del Deporte, 3(2), 7-15.

Martínez del Castillo, J. (1991). La estructura ocupacional del deporte en España. Encuesta realizada sobre los sectores de entrenamiento, docencia, animación y dirección. Madrid: CSD.

Matud, M. P. (2004). Diseño y validación de un programa de intervención psicológica con mujeres víctimas de maltrato por parte de su pareja. Informe de investigación. Madrid: Instituto de la Mujer.

Mellalieu, S. D., Neil, R., \& Hanton, S. (2006). An investigation of the mediating effects of self-confidence between anxiety intensity and direction. Research Quarterly for Sport \& Exercise, 77, 263-270.

Meyers, M. C., Bourgeois, A. E., LeUnes, A., \& Murray, N. G. (1999). Mood and psychological skills of elite and sub-elite equestrian athletes. Journal of Sport Behaviour, 22(3), 399-409.

Molero, D., Ortega, F., Caliente, I., \& Zagalaz, M. L. (2010). Estudio comparativo del 
autoconcepto físico en adolescentes en función del género y del nivel de actividad físico-deportiva. Retos. Nuevas Tendencias en Educación Física, Deporte y Recreación, 17, $38-41$.

Montero, C., Moreno-Murcia, J. A., González Ponce, I., Pulido, J. J., \& Cervelló, E. M. (2012). Ansiedad estado precompetitiva en judocas. Revista de Artes Marciales Asiáticas, 7(1), 26-43.

Montgomery, B., \& Morris, L. (1994). Living with anxiety. Singapore: Heinemann Asia.

Moraes, L. C. (1987). The relationship between anxiety and performance of Brasilian judokas. East Lansing: Michigan State University.

Moreno, J. A., Moreno, R., \& Cervelló, E. (2013). El autoconcepto físico como predictor de la intención de ser físicamente activo. Psicología y Salud, 17(2), 261-267.

Moreno, M. P., Claver, F., Gil, A., Moreno, A., \& Jiménez, R. (2014). Perfil cognitivo y emocional de los jugadores del campeonato de España de voleibol infantil y cadete 2013. Kronos, 13(2), 1-9.

Munroe, K., Hall, C., \& Fishburne, G. J. (2008). Playing with confidence: The relationship between imagery use and self-confidence and self-efficacy in youth soccer players. Journal of Sports Sciences, 26(14), 1539-1546.

Navlet, M. R. (2012). Ansiedad, estrés y estrategias de afrontamiento en el ámbito deportivo: Un estudio centrado en la diferencia entre deportes (Tesis doctoral inédita). Universidad Complutense, Madrid.

Nichols, D., \& Fines, L. (1995). Self-concept, attitude and satisfaction benefits of outdoor adventure activities: The case for recreational kayaking. Journal of Leisurability, 22(2), 1-8.

O’Brien, M., Hanton, S., \& Mellalieu, S. (2003). Individual differences, perceived control and competitive trait anxiety. Journal of Sport \& Sciences, 22, 355-356.

Parfitt, C. G., \& Pates, J. (1999). Effects of cognitive and somatic anxiety and selfconfidence on components of performance during competition. Journal of Sports Science, 17, 351-356.

Perry, J. D., \& Williams, J. M. (1998). Relation of intensity and direction of competitive trait anxiety to skill level and gender in tennis. The Sport Psychologist, 12, 169-179.

Pozo, A. (2007). Intensidad y dirección de la ansiedad competitiva y expectativas de resultados en atletas y nadadores. Revista de Psicología del Deporte, 16(2), 137-150.

Radzi, J., Yusof, S., \& Zakaria, A. (2013, junio). Pre-competition anxiety levels in individual and team sports athletes. Proceeding of the International Conference on Social Science Research, Penang, Malaysia.

Reche, C., Cepero, M., \& Rojas, F. J. (2010). Efecto de la experiencia deportiva en las habilidades psicológicas de esgrimistas del ranking nacional español. Cuadernos de Psicología del Deporte, 10(2), 33-42.

Reche, C., Cepero, M., \& Rojas, F. J. (2013). Consideraciones en el entrenamiento de habilidades psicológicas en esgrima. Cuadernos de Psicología del Deporte, 13(2), 83-88.

Robazza, C., Pellizzari, M., Bertollo, M., \& Hanin, Y. L. (2008). Functional impact of emotions on athletic performance: Comparing the IZOF model and the directional perception approach. Journal of Sports Sciences, 26(10), 1033-1047.

Rodríguez Osuna, J. (1991). Métodos de muestreo. Madrid: CIS.

Rodríguez Osuna, J. (2002). La muestra: Teoría y aplicación. En F. Alvira, M. García-Ferrando, \& J. Ibañez (Eds.), El análisis de la realidad social. Métodos y técnicas de investigación (pp. 445-482). Madrid: Alianza editorial.

Rokka, S., Mavridis, G., Bebetsos, E., \& Mavridis, K. (2009). Competitive state anxiety among junior handball players. The Sport Journal, 12(1), 1-7.

Sagar, S., \& Jowett, S. (2012). The effects of age, gender, sport type and sport level on athletes fear of failure: Implications and recommendations for sport coaches. International Journal of Coaching Science, 6(2), 61-82.

Sánchez-Alcaraz, B. J., \& Gómez, A. (2014). Autoconcepto físico en una muestra de estudiantes de primaria y su relación con el género y la práctica deportiva extraescolar. Revista de Ciencias del Deporte, 10(2), 113120. 
Scanlan, T. K., \& Passer, M. W. (1979). Sources of competitive stress in young female athletes. Journal of Sport Psychology, 1, 151-159.

Sewell, D. F., \& Edmondson, A. M. (1996). Relationships between field position and prematch competitive state anxiety in soccer and field hockey. International Journal of Sport Psychology, 27(2), 159-172.

Slater, A., \& Tiggemann, M. (2010). Uncool to do sport: A focus group study of adolescent girls' reasons for withdrawing from physical activity. Psychology of Sport \& Exercise, 11(6), 619-626. doi:10.1016/j.psychsport.201 0.07 .006

Soltani, H., Surender, K., Reza, S., Bahador, S., Hojati, Z., \& Sadat, S. (2012). Comparison of competitive state anxiety among elite and non-elite badminton players in Iran. Advances in Environmental Biology, 6(10), 2698-2703.

Tsopani, D., Dallas, G., \& Skordilis, E. K. (2011). Competitive state anxiety and performance in young female rhythmic gymnasts. Perceptual \& Motor Skills, 112, 549-560.

Vealey, R. S. (1986). Conceptualization of sport confidence and competitive orientation: Preliminary investigation and instrument development. Journal of Sport Psychology, 8, 221-246.

Vives, L., \& Garcés de Los Fayos, E. J. (2002). Autoconfianza y deporte: Aportaciones de de la psicología del deporte para su optimización. En A. Olmedilla, E. J. Garcés de Los Fayos, \& G. Nieto (Eds.), Manual de psicología del deporte (pp. 235-257). Murcia: Diego Marín.

Wadey, R. G., \& Hanton, S. (2007). The mechanisms underlying the relationship between basic psychological skills usage and competitive anxiety responses. Journal of Sport \& Exercise Psychology, 29, Sup, S210S210.

Wark, K. A., \& Witting, A. F. (1979). Sex role and sport competition anxiety. Journal of Sport Psychology, 1, 248-250.

Weinberg, R. S., \& Gould, D. (1996). Fundamentos de psicología del deporte y del ejercicio físico. Barcelona: Ariel.

Wilson, G. S., \& Raglin, J. S. (1997). Optimal and predicted anxiety in 9-12 year old track and field athletes. Scadinavian Journal of Medicine \& Science in Sports, 2, 148-152.

Revista Iberoamericana de Diagnóstico y Evaluación - e Avaliação Psicológica. RIDEP · Nº44 · Vol.2 · 158-171 2017
Woo, M. (1996). Psychological profiles of Korean elite judoists. American Journal of Sports Medicine, 24(6), 67- 71.

Woodman, T., \& Hardy, L. (2003). The relative impact of cognitive anxiety and selfconfidence upon sport performance: A metaanalysis. Journal Sports Sciences, 21(6), 443457.

Zeng, H. Z. (2003). The differences between anxiety and self-confidence between team and individual sports college varsity athletes. International Sports Journal Winter, 7(1), 2834. 